\title{
Correction to: Prenatal alcohol exposure and infant gross motor development: a prospective cohort study
}

\author{
Delyse Hutchinson 1,2,34*, George J. Youssef ${ }^{2}$, Clare McCormack', Judy Wilson', Steve Allsop ${ }^{5}$, Jake Najman ${ }^{6}$, \\ Elizabeth Elliott ${ }^{7,8}$, Lucinda Burns' ${ }^{1}$, Sue Jacobs ${ }^{9}$, Ingrid Honan', Larissa Rossen ${ }^{1}$, Hannah Fiedler ${ }^{1}$, \\ Samantha Teague ${ }^{2}$, Joanne Ryan ${ }^{4}$, Craig A. Olsson ${ }^{2,3,4,10}$ and Richard P. Mattick
}

\section{Correction to: BMC Pediatr \\ https://doi.org/10.1186/s12887-019-1516-5}

Following publication of the original article [1], the authors opted to revise two paragraphs of the article text.

Firstly, they revised the first paragraph under subsection "Characteristics associated with maternal drinking in pregnancy". Below is the updated version:

\section{Characteristics associated with maternal drinking in pregnancy}

Univariate tests compared whether abstainers and pregnancy drinkers (at any level) differed on background socio-demographics, other substance use, and physical and psychological factors (Table 3). The results show that, relative to abstainers, women who drank alcohol had greater odds of being older (e.g., 30-35 years, 1.97, 95\% CI, 1.20-3.24); completing high school $(2.61,95 \% \mathrm{CI}$, $1.48-4.61)$; having moderate $(2.29,95 \% \mathrm{CI}, 1.31-4.02)$ or high SES (4.42, 95\% CI, 2.56-7.64); being born in an English speaking country (1.88, 95\% CI, 1.33-2.66); and speaking English as their first language [2].34, 95\% CI, 1.77-3.09); and lower odds of living in a single parent household (0.61, 95\% CI, 0.39-0.95). Other factors associated with pregnancy drinking included: smoking in preg- nancy (1.67, 95\% CI, 1.18-2.36); higher estimated IQ (e.g., a score of $100-114,3.02,95 \% \mathrm{CI}, 2.01-4.53)$; and lower anxiety $(0.76,95 \% \mathrm{CI}, 0.57-0.99)$.

Secondly, they revised the first paragraph under subsection "Characteristics of women drinking in pregnancy and their partners". Please see below:

Characteristics of women drinking in pregnancy and their partners

Consistent with past research, pregnant women who consumed alcohol differed on socio-demographic characteristics compared to abstainers [36, 37]. Specifically, they were more likely to be older, tertiary educated, have moderate to high SEIFA scores (reflective of socio-economic advantage), be born in Australia or another English speaking country, and be less likely to live in a single parent household. Other factors associated with pregnancy drinking included: smoking in pregnancy; higher estimated IQ; and lower levels of anxiety. These results suggest pregnancy drinking is common among women from more affluent socio-demographic backgrounds, and among

* Correspondence: delyse.hutchinson@deakin.edu.au

${ }^{1}$ National Drug and Alcohol Research Centre, University of New South Wales,

Sydney, Australia

${ }^{2}$ Faculty of Health, School of Psychology, Centre for Social and Early

Emotional Development, Deakin University, Geelong, Victoria, Australia

Full list of author information is available at the end of the article

(c) The Author(s). 2019 Open Access This article is distributed under the terms of the Creative Commons Attribution 4.0 International License (http://creativecommons.org/licenses/by/4.0/), which permits unrestricted use, distribution, and reproduction in any medium, provided you give appropriate credit to the original author(s) and the source, provide a link to the Creative Commons license, and indicate if changes were made. The Creative Commons Public Domain Dedication waiver (http://creativecommons.org/publicdomain/zero/1.0/) applies to the data made available in this article, unless otherwise stated. 
specific at-risk groups, such as women who smoke cigarettes. Targeting these populations may result in more effective preventive intervention for pregnancy drinking.

\section{Author details}

'National Drug and Alcohol Research Centre, University of New South Wales, Sydney, Australia. ${ }^{2}$ Faculty of Health, School of Psychology, Centre for Social and Early Emotional Development, Deakin University, Geelong, Victoria, Australia. ${ }^{3}$ Murdoch Children's Research Institute, Centre for Adolescent Health, Royal Children's Hospital, Melbourne, Australia. ${ }^{4}$ Department of Paediatrics, University of Melbourne, Royal Children's Hospital, Melbourne, Australia. ${ }^{5}$ National Drug Research Institute, Curtin University, Perth, Australia. ${ }^{6}$ Queensland Alcohol and Drug Research and Education Centre and Schools of Public Health and Social Science, University of Queensland, Brisbane, Australia. ${ }^{7}$ Discipline of Child and Adolescent Health, Faculty of Medicine and Health, University of Sydney, Sydney, Australia. ${ }^{8}$ Sydney Children's Hospitals Network, Westmead, Sydney, Australia. ${ }^{9}$ Department of Obstetrics, Royal Prince Alfred Hospital, Sydney, Australia. ${ }^{10}$ School of Psychological Sciences, University of Melbourne, Melbourne, Victoria, Australia.

Published online: 04 July 2019

\section{Reference}

1. Hutchinson D, Youssef GJ, McCormack C, Wilson J, Allsop S, Najman J, Elliott E, Burns L, Jacobs S, Honan I, Rossen L, Fiedler H, Teague S, Ryan J, Olsson CA. Mattick RP. Prenatal alcohol exposure and infant gross motor development: a prospective cohort study. BMC Pediatrics. 2019;19:149 https://doi.org/10.1186/s12887-019-1516-5. 